\title{
Transanal Excision with Radiation Therapy for Rectal Adenocarcinoma
}

\author{
Nathan Tennyson, MD; William M. Mendenhall, MD; Christopher G. Morris, MS; \\ Emina H. Huang, MD; and Robert A. Zlotecki, MD, PhD
}

\begin{abstract}
Objective: To evaluate the efficacy of transanal excision (TAE) combined with radiotherapy for rectal adenocarcinoma, assess the ability of pretreatment endoscopic ultrasound (EUS) to predict failures, and determine the prognostic value of downstaging and complete pathological response.
\end{abstract}

Design: Retrospective outcomes study.

Setting: Radiation oncology clinic.

Participants: Thirty-eight patients with rectal adenocarcinoma.

Methods: The medical records of patients treated with radiotherapy from 1998 to 2008 and followed for a median of 5.9 years were reviewed.

Results: Kaplan-Meier estimates of freedom from selected endpoints at 5 years after treatment were: overall survival, 79\%; cause-specific survival, $91 \%$; local control, $90 \%$; and freedom from distant metastasis, $76 \%$. Seven patients $(21 \%)$ had eventual abdominoperineal resection or lower anterior resection, four patients had local recurrence, and three patients had incomplete treatment or poor margins. T3 lesions clinically staged by EUS were a predictor of local failure $(P=0.0110)$, but not distant metastasis $(P=0.35)$. Patients with either a pathological or clinical T3 lesion did not have a significantly greater rate of metastasis $(P=0.096)$. Patients who were downstaged did not have a significantly different rate of local recurrence or metastasis. Patients who experienced a complete pathological response did not have a significantly different rate of local control or distant metastasis.

Conclusion: Patients with early-stage rectal lesions who undergo preoperative or postoperative radiation and TAE have similar outcomes to those who undergo abdominoperineal resection; local recurrence was higher for patients with $\mathrm{T} 3$ lesions when both were compared. Abdominal surgery should be considered for these patients. TAE is reasonable when patients are unwilling or unable to tolerate the morbidity of traditional transabdominal surgery.

Keywords: Outcomes; Radiotherapy; Rectum; Transanal excision

\section{I} on transanal excision (TAE) combined with chemoradiation, both preoperatively and postoperatively, for the treatment of rectal carcinoma. Although National Comprehensive Cancer Network (NCCN) Clinical Practice Guidelines v.2.2010 recommend abdominal surgery for all but $\mathrm{T} 1$ lesions, many patients cannot tolerate the surgery, or they refuse it due to the possibility of permanent colostomy and other

Corresponding Author: Robert A. Zlotecki, MD, PhD; 2000 SW Archer Rd.; PO Box 100385; Gainesville, FL 32610-0385; Tel: (352) 265-0287; Fax: (352) 265-0759; Email: zlotera@ufl.edu complications. In these cases, a reasonable treatment alternative that prevents patient morbidity or satisfies patient requests to avoid permanent colostomy is needed.

A few small, published series have shown that preoperative chemoradiation can effectively downstage selected $\mathrm{T} 2$ and $\mathrm{T} 3$ rectal lesions that might have required abdominoperineal resection (APR) so they can be subsequently excised and

Received: December 16, 2011

Revised: April 27, 2012

Accepted: June 20, 2012

doi: $10.3|2| / \mathrm{cmr} .2012 .1072$ 
managed by TAE, thereby preserving sphincter function. ${ }^{2-5}$ Postoperative chemoradiotherapy is a reasonable option for patients who were thought to have T1 lesions, but then on pathology, had either a higher stage lesion or unfavorable histology. ${ }^{6} \mathrm{NCCN}$ guidelines currently recommend abdominal surgery for these patients, but patients might decline this option for a number of reasons. The purpose of this study was to report the outcomes of patients with rectal adenocarcinoma treated with radiation therapy and TAE at the University of Florida between July 1998 and March 2008. Additionally, we assessed the power of endoscopic ultrasound (EUS) staging, pathologic downstaging, and pathological response for predicting local recurrence and distant metastasis.

\section{Methods}

Under an institutional review board-approved protocol, we reviewed the medical records of 38 patients treated with preoperative or postoperative radiation and TAE for rectal adenocarcinoma at the University of Florida between July of 1998 and March of 2008. All patients had invasive carcinoma. This case series represented a population of patients who were either poor candidates for conventional open surgery due to age, infirmity, medical comorbidities, or a combination of these factors. The patients specifically declined conventional surgical resection either due to overall risk or refusal to consider the potential consequence of loss of sphincter function and the requirement of a colostomy. The median observed length of follow-up was 5.9 years (range, 0.3 to 11.1 years). All patients had adequate preoperative workup to exclude extrapelvic disease. These tests include computed tomography (CT) scans of the abdomen and pelvis, chest CT or X-ray, complete blood count, liver function tests, and carcinoembryonic antigen levels. Most patients $(n=32)$ also received EUS for preoperative staging. Patients were staged according to the $7^{\text {th }}$ edition of the American Joint Committee on Cancer's (AJCC) Cancer Staging Manual. ${ }^{7}$ Those who did not have EUS were not included in our clinical staging analysis, but were included in the analysis for the other study endpoints.

Radiotherapy treatment typically was delivered using 18-MV photons on the lateral and anterior portals and 6-MV photons for the posterior field. The dose was specified at an isodose line that encompassed the primary tumor and regional lymph nodes. All patients were treated with the 3- or 4-field technique. Twenty-six patients were treated with preoperative radiotherapy using 4500 cGy with a 3-field boost to 5040 cGy. One patient was treated to $4860 \mathrm{cGy}$, and four were treated to $4500 \mathrm{cGy}$. Postoperative patients were treated to various doses. One patient was treated to $5040 \mathrm{cGy}$, one patient to $5300 \mathrm{cGy}$, two patients to $5580 \mathrm{cGy}$, and one patient to 5940 cGy. One patient, who chose to stop her radiotherapy treatment, received $3240 \mathrm{cGy}$.

Concomitant chemotherapy was given to 26 of 32 preoperative patients during radiotherapy treatment. Twenty-three patients were treated with fluorouracil (5FU) by continuous infusion (although two did not complete 5FU due to complications), three were treated with capecitabine. Two of the six postoperative radiotherapy patients were treated with $5 \mathrm{FU}$.

For patients who had preoperative radiotherapy, TAE was performed on average 48 days after completing radiation therapy (range, 28 to 87 days). The primary factors related to variability in surgical timing were patient tolerance and willingness to undergo surgical resection. Postoperative radiotherapy was started a median 32 days after surgery (range, 27 to 48 days). This range was less variable due to overall rapid and consistent recovery from the minimally invasive surgical TAR procedure. Close margins were defined as those closer than $5 \mathrm{~mm}$ of the inked margin. There were no instances of residual positive surgical margins on final pathologic review. Surgical technique has been described in a previous publication. ${ }^{8}$

Study endpoints included local control, distant metastasis, cause-specific survival, and toxicity. Additionally, the prognostic value of both complete pathological response and clinical staging through EUS ultrasound was examined. Toxicity was measured by both the Radiation Therapy Oncology Group guidelines and the National Cancer Institute Common Terminology Criteria for Adverse Events, version 4.0. ${ }^{9}$ Radiation complications were most often found in the visits immediately following the completion of radiation, but any side effect that could reasonably be attributed to radiation was coded.

All statistical computations were performed using SAS software (SAS Institute, Cary, NC). The Kaplan-Meier product-limit method was used to estimate freedom from specified endpoints. ${ }^{10}$ The log-rank test statistic was used to detect significant differences in these endpoints between strata of selected prognostic factors.

\section{Results}

Patients and tumor characteristics are described in table 1. After surgery, patients who underwent preoperative radiotherapy were staged pathologically utilizing the AJCC yp designator to account for the effect of neoadjuvant therapy as follows: 12 had ypCR; 5 had ypT1; 12 had ypT2; and 2 had ypT3 lesions. The pathology staging among patients undergoing postoperative radiation therapy was distributed as follows: 2 pT1; 2 pT2; and 2 pT3 lesions. One patient in the preoperative group had no pathology reported due to death shortly following surgery. Table 2 shows a comparison of EUS staging and final pathological staging for patients undergoing preoperative radiation therapy.

Local-regional control was achieved in all of the postoperative patients and in 28 of the 32 preoperative patients (88\%). Four patients had an initial local recurrence; 3 of 4 patients with a local recurrence were staged clinically as T3 by EUS, and the other patient with a local recurrence did not have an EUS for pretreatment staging. Of the four patients who had local 
Table 1. Patient and tumor characteristics.

\begin{tabular}{|c|c|}
\hline Characteristic & Value \\
\hline \multicolumn{2}{|l|}{ Gender (N=38) } \\
\hline Male (n) & 26 \\
\hline Female (n) & 12 \\
\hline Age, mean (y) & 63 (range, 41-86) \\
\hline Tumor size, mean $(\mathrm{cm})$ & 3.1 (range, 2-5) \\
\hline \multicolumn{2}{|c|}{ EUS clinical stage for pre-op RT patients $(\mathrm{N}=32)$} \\
\hline T1N0 (n) & 5 \\
\hline T2NO (n) & 11 \\
\hline T3N0 (n) & 9 \\
\hline T2N1 (n) & 1 \\
\hline T3N1 (n) & 2 \\
\hline Not performed (n) & 4 \\
\hline \multicolumn{2}{|c|}{ EUS clinical stage for post-op RT patients $(\mathrm{N}=6)$} \\
\hline T1No (n) & 2 \\
\hline T2NO (n) & 2 \\
\hline Not performed $(n)$ & 2 \\
\hline \multicolumn{2}{|l|}{ Pretreatment differentiation ( $\mathrm{N}=38$ ) } \\
\hline Well/moderate (n) & 21 \\
\hline Poor $(n)$ & 1 \\
\hline Carcinoma in situ (n) & 6 \\
\hline No data (n) & 10 \\
\hline Distance from the anal verge, mean $(\mathrm{cm})$ & 5.7 (range, 0.5-15) \\
\hline
\end{tabular}

recurrence, one underwent palliative fulguration due to age and previous development of a metastasis. The other three patients were salvaged with APR, and one eventually developed a metastasis. In addition to those with a local recurrence, three other patients underwent eventual APR or lower anterior resection (LAR). One postoperative patient had an APR after refusing to continue with radiation treatment. One preoperative patient had an APR due to close margins and poor histological characteristics of the specimen. Another preoperative patient had an LAR due to close margins observed on the TAE pathologic specimen. These three patients were disease-free after surgery. Seven preoperative radiotherapy patients developed a metastasis, two of whom also failed locally. One patient in the postoperative radiotherapy group developed a metastasis.

Overall and cause-specific survival rates at 5 years were $79 \%$ and $91 \%$, respectively. Twenty-two patients (58\%) were free of distant metastases, salvage surgery, local recurrence, and grade 3 complications at 5 years. Patients with T3 lesions clinically staged on EUS tended to fail locally (figure 1), but not distantly. Patients with either a pathological stage T3 or clinical stage T3 lesion did not have a significantly greater rate of metastasis than the other patients. Patients who were downstaged did not have a significantly different rate of metastasis or local recurrence. Patients who experienced a complete pathological response did not have a significantly different rate of local control or distant metastasis than those with a partial response.

Significant toxicity included two cases of grade 3 diarrhea and three cases of dehydration requiring parenteral support. Grade 3 complications were experienced by 5 patients $(13 \%)$, grade 1 or 2 complications by 21 patients $(55 \%)$, and no radiation complications by 12 patients $(32 \%)$. One patient experienced permanent erectile dysfunction. All patients who underwent TAE only (and not LAR or APR) had preserved longterm continence.

\section{Discussion}

Current NCCN guidelines recommend TAE only for T1 lesions, and abdominal resection recommended for the remainder of patients. ${ }^{1}$ The largest randomized trials of rectal cancer generally have used radical abdominal surgery with good results. ${ }^{11-14}$ To our knowledge, there have not been large randomized trials comparing TAE and preoperative chemoradiotherapy to radical abdominal surgery. However, quality of life issues associated with radical abdominal

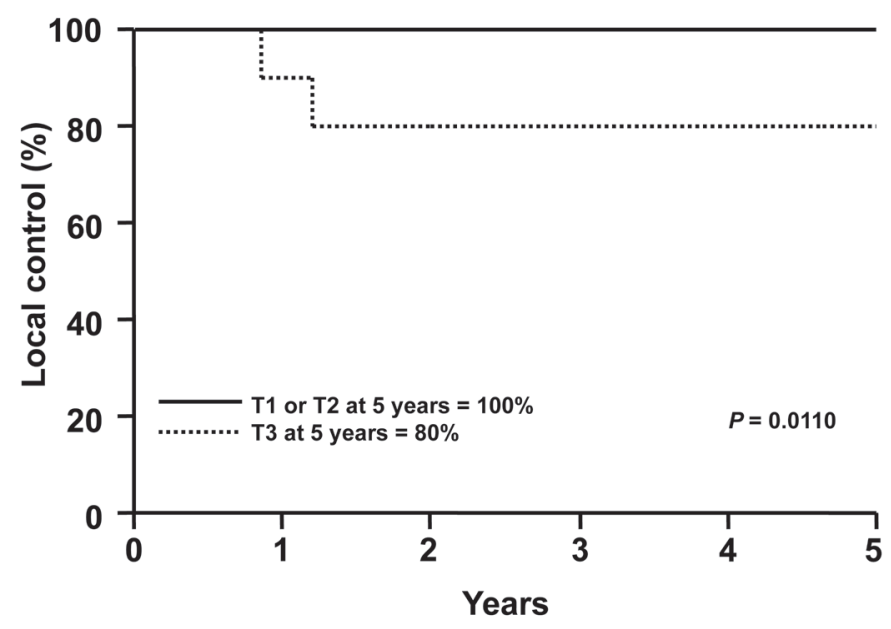

Figure 1. Local control rates for all patients with endoscopic ultrasound staging. 
Table 2. EUS T stage and final pathology for patients undergoing preoperative RT and EUS staging.

\begin{tabular}{cc}
\hline Final Pathology by EUS stage & N \\
\hline T1 $(n=5)$ & 4 \\
ypCR & 1 \\
урT1 & 0 \\
урT2 & 0 \\
урT3 & \\
T2 (n=12) & \\
урCR & 3 \\
урT1 & 4 \\
ypT2 & 4 \\
ypT3 & 1 \\
T3 (n=11) & \\
ypCR & \\
ypT1 & 3 \\
ypT2 & 0 \\
урT3 & 7 \\
\hline
\end{tabular}

EUS, endoscopic ultrasound; RT, radiation therapy

surgery provides the motivation to use TAE to treat rectal cancer. Varpe et a ${ }^{15}$ studied the quality of life for patients who had undergone LAR and APR. Patients who were alive without signs of recurrence $(n=65)$ answered the same questionnaires before surgery and 1 year after surgery. The study showed that urinary incontinence and other complications worsened social function following abdominal surgery, whether the patient had an LAR or APR. Additionally, sexual functioning in men was affected, often with permanent damage.$^{15} \mathrm{TAE}$, on the other hand, has a very low complication rate. Doornebosch et $\mathrm{al}^{16}$ studied 50 patients, issuing them a quality of life survey before and after TAE. They showed that quality of life actually improves after patients have TAE. ${ }^{16}$ Our study reflects this finding and shows that although there are some complications potentially related to increased intensity of therapy, in general, patients treated with TAE and radiation do well with little long-term sequelae.

This improvement in quality of life with TAE versus abdominal resection has inspired an increasing number of studies comparing TAE and chemoradiation to more extensive rectal cancer surgery techniques. However, it is important to distinguish patients who would be good candidates for TAE from those who would be better served by traditional therapy. NCCN guidelines currently differentiate treatment regimens for rectal cancer based largely on AJCC staging. Depth of invasion, nodal status, and presence of distant metastasis are very important prognostic variables that should be used as part of the decision-making process. However, histology, response to therapy, and DNA methylation patterns have been shown to be prognostic and, therefore, can be used to help identify patients with $\mathrm{T} 2$ or $\mathrm{T} 3 \mathrm{~N} 0$ lesions who might be good candidates for TAE.

The use of TAE as an alternative to abdominal surgery is dependent on the ability of EUS to accurately stage rectal cancer. Puli et a $\mathbf{l}^{17}$ performed a meta-analysis of 42 studies to determine the pooled sensitivity and specificity of EUS. The sensitivity and specificity to identify $\mathrm{T}$ stage for these cancers was above $85 \%$ for $\mathrm{T} 1$ to $\mathrm{T} 3$ stage rectal cancer. ${ }^{17}$ In our series, we found that EUS staging was predictive of local failure, but not distant metastasis. The accuracy of EUS allows patients with early-stage rectal cancer to be identified and the possibility of increased risk for local recurrence associated with $\mathrm{T} 2$ or greater stage after transanal endoscopic microsurgery to be more thoroughly evaluated and reconsidered.

Tumor size, distance from the anal verge, and histological characteristics are important prognostic variables when considering a patient for TAE. In a prospective randomized trial of 70 patients with rectal cancer treated for $\mathrm{T} 2 \mathrm{~N} 0$ lesions, Lezoche et $\mathrm{al}^{4,5}$ found that total mesorectal excision and TAE with preoperative chemoradiation resulted in similar outcomes at the patients' 5-year follow-up. In this study, the patients selected had tumors located within $6 \mathrm{~cm}$ of the anal verge, with diameters $<3 \mathrm{~cm}$, and of well- or moderatelydifferentiated histology. Also, patients with neural, vessel, or lymphatic invasion were excluded. Thus, the study showed that patients with favorable tumor characteristics can have a TAE without worsening their outcomes.

Clinical and pathological response to radiotherapy can be used as a prognostic tool to identify patients who are at higher risk for distant and local failure. In a study of 196 patients with $\mathrm{T} 1$ to $\mathrm{T} 3$ rectal cancer, Guerrieri et $\mathrm{al}^{3}$ showed that TAE with preoperative radiotherapy results in similar survival and local recurrence outcomes when compared to transanal endoscopic microsurgery outcomes found in the literature. ${ }^{3}$ The study also showed that patients who respond either clinically or pathologically to preoperative therapy tend to do well. They found no local recurrences or distant metastases for any downstaged or downsized tumors, suggesting that response to therapy is prognostic in patients who have had TAE.

Treatment decisions can be reasonably based on clinical or pathological response to therapy due to its prognostic value. Nair et $\mathrm{al}^{18}$ conducted a study where patients who had preoperative chemoradiotherapy followed by transanal excision were offered more extensive surgery (APR or LAR) if they did not have a complete pathological response. The goal of this treatment regimen was to treat patients who tend to do poorly more aggressively. They found that overall 5 -year survival rates for patients with T2/T3N0 and T2/T3N1 lesions were $84 \%$ and $81 \%$, respectively, which are similar to historic controls. ${ }^{18}$ 
Using tumor markers to predict recurrence rates is a promising way to determine which patients would be good candidates for TAE. De Maat et al ${ }^{19}$ attempted to locate prognostically significant tumor methylation patterns by taking pathological specimens from 325 non-irradiated patients who underwent resection for rectal cancer and identifying methylation clusters associated with local recurrence rates. The study found that these different clusters had significantly different local recurrence rates. Thus, methylation pattern serves as another prognostic tool for determining whether a patient is a potential candidate for transanal excision. ${ }^{19}$

Taken together, these studies elucidate a reasonable treatment approach for early-stage rectal cancer. Patients with small well- or moderately-differentiated tumors with a "good" DNA methylation pattern could be initially selected for TAE. Patients would then undergo preoperative chemoradiotherapy, and those who did not respond either clinically or pathologically after TAE could be offered additional surgery or chemotherapy. This treatment-decision process would hopefully best select those patients with low-risk cancer who could be spared an abdominal resection.

More large randomized studies with well-defined selection criteria are needed to examine the question of whether TAE combined with preoperative chemoradiotherapy is an equivalent treatment to traditional therapy. There are currently no large randomized studies evaluating this issue. However, NCCN ACOSOG Z 6041 is a phase II trial in which rectal cancer patients undergo high-dose external-beam radiotherapy and receive oral capecitabine and oxaliplatin followed by TAE for T2N0 lesions. The goal is to enroll 100 patients, and it is due to finish in 2014. Although not randomized, it will be the largest study in the United States evaluating the use of TAE with preoperative chemoradiotherapy for T2N0 lesions. If this study shows promising results, it may promote further studies and the possible change of NCCN guidelines.

\section{Conclusion}

Our analysis shows that patients with early-stage rectal lesions who undergo preoperative or postoperative radiation and TAE have similar survival outcomes to similar patients in the published results who undergo abdominal resection. Local recurrence was higher for patients with T3 lesions when compared to historic controls, and these patients should be considered for total mesorectal excision. Complications from TAE are less morbid than the comparable radical surgery. Complete pathological response and downstaging were not statistically significant predictive clinicopathologic features, although the small sample size combined with good overall survival rates makes it difficult to achieve statistical significance. Given our survival endpoints, TAE is a reasonable option when the patient is unwilling or unable to tolerate the morbidity associated with traditional transabdominal surgery.

\section{Acknowledgements}

The authors would like to thank Jessica Kirwan and the staff in the research office at the Department of Radiation Oncology at the University of Florida for helping edit and prepare the manuscript.

\section{References}

1. NCCN clinical practice guidelines in oncology - v.2.2010. National Comprehensive Cancer Network 2010. Available at: http://www.nccn.org/professionals/physician_gls/f_ guidelines.asp. Accessed December 7, 2010.

2. Callender GG, Das P, Rodriguez-Bigas MA, Skibber JM, Crane $\mathrm{CH}$, Krishnan S, Delclos ME, Feig BW. Local excision after preoperative chemoradiation results in an equivalent outcome to total mesorectal excision in selected patients with $\mathrm{T} 3$ rectal cancer. Ann Surg Oncol 2010;17:441-447.

3. Guerrieri M, Baldarelli M, Organetti L, Grillo Ruggeri F, Mantello G, Bartolacci S, Lezoche E. Transanal endoscopic microsurgery for the treatment of selected patients with distal rectal cancer: 15 years experience. Surg Endosc 2008;22:2030-2035.

4. Lezoche E, Guerrieri M, Paganini AM, D’Ambrosio G, Baldarelli M, Lezoche G, Feliciotti F, De Sanctis A. Transanal endoscopic versus total mesorectal laparoscopic resections of T2-N0 low rectal cancers after neoadjuvant treatment: a prospective randomized trial with a 3-years minimum follow-up period. Surg Endosc 2005;19:751-756.

5. Lezoche G, Baldarelli M, Guerrieri M, Paganini AM, De Sanctis A, Bartolacci S, Lezoche E. A prospective randomized study with a 5-year minimum follow-up evaluation of transanal endoscopic microsurgery versus laparoscopic total mesorectal excision after neoadjuvant therapy. Surg Endosc 2008;22:352-358.

6. Mendenhall WM, Morris CG, Rout WR, Zlotecki RA, Lind DS, Hochwald SN, Schell SR, Copeland EM 3rd. Local excision and postoperative radiation therapy for rectal adenocarcinoma. Int J Cancer 2001;96 Suppl:89-96.

7. American Joint Committee on Cancer: AJCC Cancer Staging Handbook. $7^{\text {th }}$ ed. New York, NY: Springer; 2010.

8. Meadows K, Morris CG, Rout WR, Zlotecki RA, Hochwald SN, Marsh RD, Copeland EM, Mendenhall WM. Preoperative radiotherapy alone or combined with chemotherapy followed by transanal excision for rectal adenocarcinoma. Am J Clin Oncol 2006;29:430-434.

9. Common Terminology Criteria for Adverse Events (CTCAE) v4.0. Cancer Therapy Evaluation Program web site. Available at http://ctep.cancer.gov/protocolDevelopment/electronic applications/ctc.htm\#ctc_40. Accessed June 4, 2010.

10. Kaplan EL, Meier P. Nonparametric estimation from incomplete observations. J Am Stat Assoc 1958;53:457-481.

11. Improved survival with preoperative radiotherapy in resectable rectal cancer. Swedish Rectal Cancer Trial. N Engl J Med 1997;336:980-987.

12. Kapiteijn E, Marijnen CA, Nagtegaal ID, Putter H, Steup WH, Wiggers T, Rutten HJ, Pahlman L, Glimelius B, van Krieken JH, Leer JW, van de Velde CJ; Dutch Colorectal Cancer Group. Preoperative radiotherapy combined with total mesorectal excision for resectable rectal cancer. N Engl J Med 2001;345:638-646.

13. Law WL, Chu KW. Anterior resection for rectal cancer with mesorectal excision: a prospective evaluation of 622 patients. Ann Surg 2004;240:260-268. 
14. Gunderson LL, Sargent DJ, Tepper JE, Wolmark N, O'Connell MJ, Begovic M, Allmer C, Colangelo L, Smalley SR, Haller DG, Martenson JA, Mayer RJ, Rich TA, Ajani JA, MacDonald JS, Willett CG, Goldberg RM. Impact of T and $\mathrm{N}$ stage and treatment on survival and relapse in adjuvant rectal cancer: a pooled analysis. J Clin Oncol 2004;22: $1785-1796$.

15. Varpe P, Huhtinen H, Rantala A, Salminen P, Rautava P, Hurme S, Grönroos J. Quality of life after surgery for rectal cancer with special reference to pelvic floor dysfunction. Colorectal Dis 2011;13:399-405.

16. Doornebosch PG, Gosselink MP, Neijenhuis PA, Schouten WR, Tollenaar RA, de Graaf EJ. Impact of transanal endoscopic microsurgery on functional outcome and quality of life. Int $\mathrm{J}$ Colorectal Dis 2008;23:709-713.

17. Puli SR, Bechtold ML, Reddy JB, Choudhary A, Antillon MR, Brugge WR. How good is endoscopic ultrasound in differentiating various $\mathrm{T}$ stages of rectal cancer? Meta-analysis and systematic review. Ann Surg Oncol 2009;16:254-265.

18. Nair RM, Siegel EM, Chen DT, Fulp WJ, Yeatman TJ, Malafa MP, Marcet J, Shibata D. Long-term results of transanal excision after neoadjuvant chemoradiation for T2 and T3 adenocarcinomas of the rectum. J Gastrointest Surg 2008;12:1797-805; discussion 805-806.

19. de Maat MF, van de Velde CJ, Benard A, Putter H, Morreau H, van Krieken JH, Meershoek Klein-Kranenbarg E, de Graaf EJ, Tollenaar RA, Hoon DS. Identification of a quantitative MINT locus methylation profile predicting local regional recurrence of rectal cancer. Clin Cancer Res 2010;16: $2811-2818$

\section{Author Affiliations}

Nathan Tennyson, MD*; William M. Mendenhall, MD*; Christopher G. Morris, MS*; Emina H. Huang, $M D^{*}$;

Robert A. Zlotecki, $M D, P h D^{*}$

*Department of Radiation Oncology, College of Medicine, University of Florida, Gainesville, FL, USA

Department of Surgery, College of Medicine,

University of Florida, Gainesville, FL, USA 